\title{
High-resolution, real-time exercise stress cine
}

\author{
Rizwan Ahmad ${ }^{1}$, Samuel T Ting ${ }^{1 *}$, Jason Craft ${ }^{1}$, Shivraman Giri' ${ }^{2}$ Ning Jin ${ }^{2}$, Subha V Raman ${ }^{1}$, Orlando P Simonetti ${ }^{1}$
}

From 17th Annual SCMR Scientific Sessions

New Orleans, LA, USA. 16-19 January 2014

\section{Background}

Despite the technological advances made in the field of MRI, further improvements in both data acquisition and processing are required to expand the reliability and diagnostic accuracy of challenging CMR applications such as real-time stress imaging. Due to exaggerated breathing motion and high heart rates, real-time stress images are limited in terms of resolution and often exhibit significant artifacts. In this work, we combine a recently proposed method for variable density incoherent spatiotemporal sampling, called VISTA [1], and SPIRiT-based reconstruction [2] with 3D spatiotemporal regularization to reconstruct real-time stress cine images.

\section{Methods}

Rest and stress (Bruce protocol) cine images were acquired from three healthy volunteers using a $1.5 \mathrm{~T}$ (Avanto, Siemens) scanner with 32-channel body coil array. The acquisition was carried out under free-breathing conditions in both short and long axis orientations. The data were collected using four acceleration rates $(R=4,6,8$, and 10) and two sampling patterns: traditional uniform interleaved sampling (UIS), and VISTA. Other imaging parameters included: 48 frames, $192 \times 128$ matrix size, $360 \times 292 \mathrm{~mm} 2$ FOV, $8 \mathrm{~mm}$ slice thickness, and maximum temporal resolution of $32 \mathrm{~ms}$ (at rate 10). For VISTA sampling, a nonlinear conjugate gradient method was used to perform SPIRiT-based reconstruction, with the SPIRiT kernels estimated from the fully-sampled, time-averaged data. To exploit the spatiotemporal structure in the image, 11-regularization in the 3D discrete wavelet domain was employed. All data were reconstructed offline in MATLAB (version 2013b) using an Intel Core i5 workstation with 24 GB memory. Signal to noise ratio (SNR)-defined as the inverse of the standard deviation of pixel intensities over peripheral regions devoid of NMR signal-was also measured.

\footnotetext{
${ }^{1}$ Ohio State University, Columbus, Ohio, USA
}

Full list of author information is available at the end of the article

\section{Results}

Figure 1 shows example images at acceleration rates of 4 and 10 using the traditional UIS sampling and GRAPPA

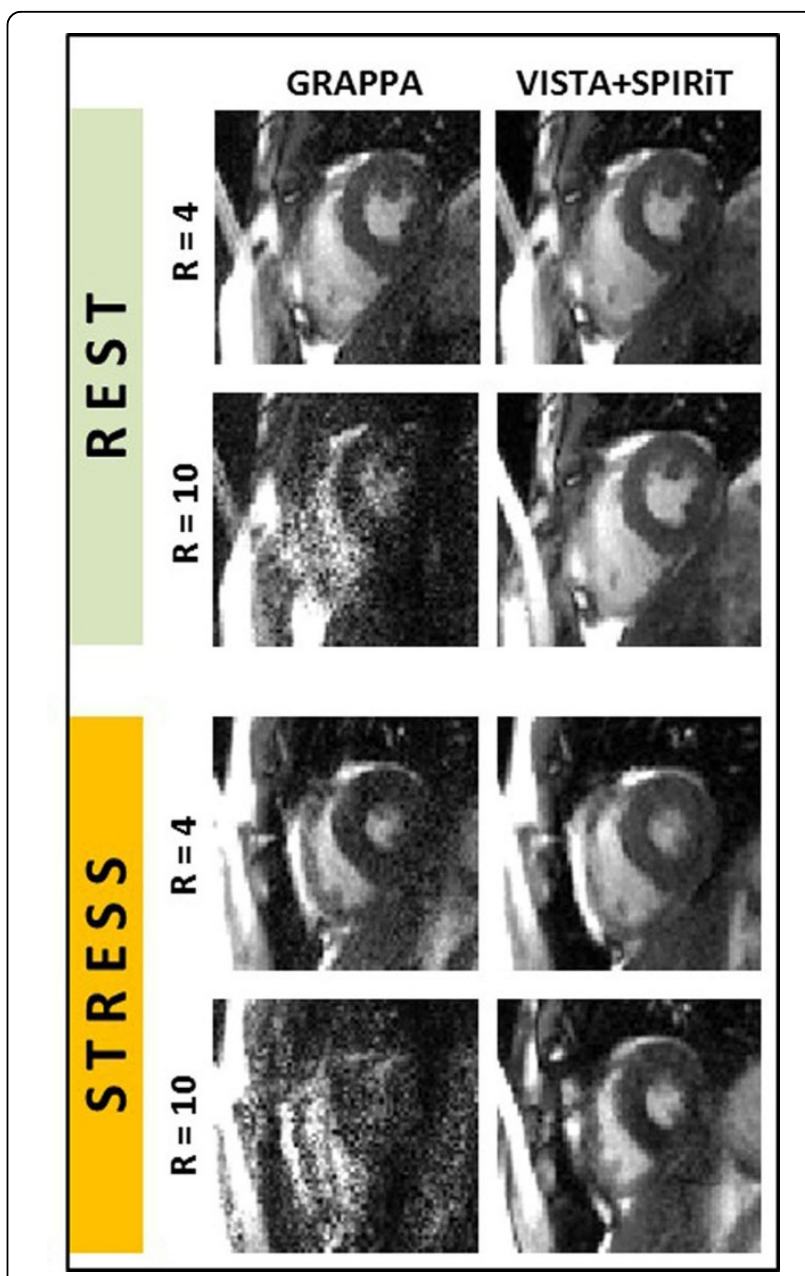

Figure 1 Real-time, free-breathing cine images under rest and stress conditions. The proposed VISTA+SPIRiT is compared against traditional GRAPPA reconstruction; the results at two different acceleration rates are displayed. 
reconstruction (UIS+GRAPPA) and the incoherent VISTA sampling and 11-regularized SPIRiT (VISTA+SPIRiT). Compared to the traditional UIS+GRAPPA approach, VISTA+SPIRiT results showed 3 to 5 -fold improvement in SNR. Visually, VISTA+SPIRiT also shows minimum artifacts compared to UIS+GRAPPA, especially at high acceleration rates. For VISTA+SPIRiT, the reconstruction time for one dataset was under 30 minutes.

\section{Conclusions}

Combination of VISTA and SPIRiT with spatiotemporal 11-regularization allows high quality images at sub $35-\mathrm{ms}$ temporal resolution and sub $2.5-\mathrm{mm}$ spatial resolution under conditions of high heart rates and exaggerated breathing motion following exercise stress.

\section{Funding}

The work was funded by R01 HL102450 from the NIH.

\section{Authors' details}

'Ohio State University, Columbus, Ohio, USA. ${ }^{2}$ Siemens Healthcare,

Columbus, Ohio, USA.

Published: 16 January 2014

\section{References}

1. Ahmad R, et al: ISMRM DSIR Workshop 2013.

2. Lustig M, et al: Mag Res Med 2010, 64(2):457-71.

doi:10.1186/1532-429X-16-S1-W14

Cite this article as: Ahmad et al:: High-resolution, real-time exercise stress cine. Journal of Cardiovascular Magnetic Resonance 2014 16(Suppl 1): W14
Submit your next manuscript to BioMed Central and take full advantage of:

- Convenient online submission

- Thorough peer review

- No space constraints or color figure charges

- Immediate publication on acceptance

- Inclusion in PubMed, CAS, Scopus and Google Scholar

- Research which is freely available for redistribution

Submit your manuscript at www.biomedcentral.com/submit 